\title{
Adverse Cardiometabolic Outcomes in Obese Patients Correlates Strongly with Defective Branched-chain Amino Acid Catabolism
}

Dipsikha Biswas, Andrew Cowie, Kathleen Tozer, Lester J. Perez, Purvi Trivedi, Jordan J. Bartlett, Luke Duffley, Khoi Thien Dao, Geena V. Paramel, Christie Aguiar, Alexandra M. Yip, Jennifer Shea, Keith Brunt, Jean-Francois Legare, Ansar Hassan, Petra Kienesberger, Thomas Pulinilkunnil.

Correspondence author: Dipsikha Biswas

DOI: https://doi.org/10.1016/j.yjmcc.2018.07.113

Background: Dysregulated branched amino acid (BCAA) metabolism is an important predictor of impaired insulin sensitivity and cardiovascular dysfunction. BCAA uptake is facilitated by branch chain aminotransferase (BCAT) to yield branched-chain $\alpha$-keto acids (BCKA). Mitochondrial oxidation of BCKA is catalyzed by branched chain ketoacid dehydrogenase (BCKDH), enzyme sensitive to inhibitory phosphorylation by BCKD kinase. However, the clinical association between dysregulated BCAA catabolism and cardiovascular dysfunction merits investigation. 